\title{
Mitochondrial DNA copy number and trimethylamine levels in the blood: New insights on cardiovascular disease biomarkers
}

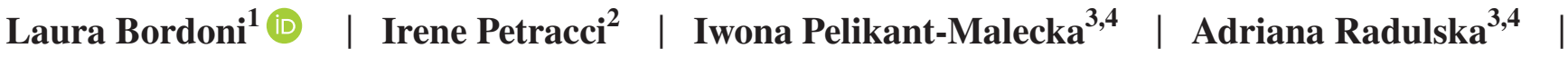 Marco Piangerelli $^{5}$ (D) | Joanna J. Samulak ${ }^{6}$ | Lukasz Lewicki $^{7}$ | Leszek Kalinowski ${ }^{3,4,8}$ | Rosita Gabbianelli $^{1}$ (i) | Robert A. Olek $^{9}$ (i)}

${ }^{1}$ Unit of Molecular Biology and Nutrigenomics, School of Pharmacy, University of Camerino, Camerino, Italy

${ }^{2}$ School of Advanced Studies, University of Camerino, Camerino, Italy

${ }^{3}$ Division of Medical Laboratory Diagnostics, Medical University of Gdansk, Gdansk, Poland

${ }^{4}$ Biobanking and Biomolecular Resources Research Infrastructure Poland (BBMRI.PL), Gdansk, Poland

${ }^{5}$ Computer Science Division and Mathematics Division, School of Science and Technology, University of Camerino, Camerino, Italy

${ }^{6}$ Doctoral School, Gdansk University of Physical Education and Sport, Gdansk, Poland

${ }^{7}$ Department of Cardiology and Angiology, Kashubian Center for Heart and Vascular Diseases, Pomeranian Hospitals, Wejherowo, Poland

${ }^{8}$ Department of Mechanics of Materials and Structures, Gdansk University of Technology, Gdansk, Poland

${ }^{9}$ Department of Athletics, Strength and Conditioning, Poznan University of Physical Education, Poznan, Poland

\section{Correspondence}

Laura Bordoni, Unit of Molecular Biology,

School of Pharmacy, University of

Camerino, Camerino, 62032, Italy.

Email: laura.bordoni@unicam.it

\section{Funding information}

Funding for reagents were provided by Prof. Rosita Gabbianelli (FPA000033), and Ministry of Science and Higher Education, Poland, grant no. DIR/WK/2017/01

\begin{abstract}
Among cardiovascular disease (CVD) biomarkers, the mitochondrial DNA copy number (mtDNAcn) is a promising candidate. A growing attention has been also dedicated to trimethylamine-N-oxide (TMAO), an oxidative derivative of the gut metabolite trimethylamine (TMA). With the aim to identify biomarkers predictive of CVD, we investigated TMA, TMAO, and mtDNAcn in a population of 389 coronary artery disease (CAD) patients and 151 healthy controls, in association with established risk factors for CVD (sex, age, hypertension, smoking, diabetes, glomerular filtration rate $[\mathrm{GFR}]$ ) and troponin, an established marker of CAD. MtDNAcn was significantly lower in CAD patients; it correlates with GFR and TMA, but not with TMAO. A biomarker including mtDNAcn, sex, and hypertension (but neither TMA nor TMAO) emerged as a good predictor of CAD. Our findings support the mtDNAcn as a promising plastic biomarker, useful to monitor the exposure to risk factors and the efficacy of preventive interventions for a personalized CAD risk reduction.
\end{abstract}

\footnotetext{
Abbreviations: ACS, acute coronary syndrome; ATP, adenosine triphosphate; BMI, body mass index; CAD, coronary artery disease; CVD, cardiovascular disease; HS, high severity; MS, mild severity; mtDNA, mitochondrial DNA; mtDNAcn, mitochondrial DNA copy number; nDNA, nuclear DNA; NUMT, nuclear mitochondrial DNA sequence; SA, stable angina; TMA, trimethylamine; TMAO, trimethylamine-N-oxide.

Rosita Gabbianelli and Robert A. Olek contributed equally to this study.

This is an open access article under the terms of the Creative Commons Attribution-NonCommercial License, which permits use, distribution and reproduction in any medium, provided the original work is properly cited and is not used for commercial purposes.

(c) 2021 The Authors. The FASEB Journal published by Wiley Periodicals LLC on behalf of Federation of American Societies for Experimental Biology.
} 


\section{K E Y W O R D S}

biomarkers, cardiovascular disease, hypertension, mitochondrial DNA, sex, TMAO

\section{1 | INTRODUCTION}

Cardiovascular disease (CVD) is a major cause of death and disability worldwide. ${ }^{1}$ The primary prevention of CVD relies on the identification of high-risk individuals before the manifestation of the event. Thus, the need for new methods for accurate risk stratification is drawing attention, and several biomarkers have been proposed to predict cardiovascular events. ${ }^{2}$ Biomarkers play a critical role in the definition, prognosis, and decision-making in cardiovascular disease management. Nevertheless, despite increasing efforts, the possibility to identify high-risk individuals is still limited. Among the novel candidates, trimethylamine $\mathrm{N}$-oxide (TMAO) has attracted a growing attention as a potential promoter of atherosclerosis in humans. ${ }^{3-7}$ TMAO is the oxidative metabolite of trimethylamine (TMA), which is produced by the gut microbiome from dietary precursors (ie, choline, betaine, and carnitine). The TMA is absorbed via the intestinal epithelium and it is further oxidized in the liver to TMAO, which is then excreted in urine. Several hypotheses have linked the TMAO to the development of atherosclerosis and CVD risk via promotion of platelet hyper-reactivity, ${ }^{8}$ pro-inflammatory changes in the artery wall, ${ }^{9}$ enhanced macrophage cholesterol accumulation and foam cell formation, ${ }^{3}$ increased levels of pro-inflammatory monocytes. ${ }^{10}$ Nevertheless, contrasting evidence on the association of TMAO and CVD emerged, ${ }^{11-}$ 16 and a clear mechanistic explanation of this association is still missing.

Another interesting peripheral biomarker that has been recently proposed for CVD prediction is the mitochondrial DNA copy number (mtDNAcn). ${ }^{17}$ Human mitochondrial DNA (mtDNA) is a small, circular, and multi-copy genome, located in the inner matrix of mitochondria. It incorporates 37 mitochondrial genes (13 coding for essential components of the mitochondrial electron transport chain and of the ATP synthase complex, 22 for mitochondrial transfer RNAs and 2 for ribosomal RNAs). Mitochondrial DNA content reflects the energy demand of a cell ${ }^{18}$ and is disturbed by imbalanced energy metabolism and reactive oxygen species overproduction. Thus, mtDNAcn changes have been proposed as an early biomarker of damage and mitochondrial dysfunction. ${ }^{19,20}$ Remarkably, since mtDNAcn changes have been associated with both intrinsic and extrinsic factors, ${ }^{21,22}$ mtDNAcn has been proposed as a potential biomarker for complex diseases, which are linked to both genetics and environmental exposures. ${ }^{23}$

Circulating mtDNAcn has been investigated in cardiovascular diseases ${ }^{24}$ mainly analyzing peripheral blood cells .

\section{Highlights}

- mtDNAcn measured in whole blood is associated to the cardiovascular health status in humans;

- mtDNAcn is reduced in CAD and hypertension, and inversely correlates with GFR;

- mtDNA, sex, and hypertension together represent a good predictive biomarker for CAD;

- TMA metabolism is different in healthy subjects and CAD patients;

- TMA and TMAO are not good predictors of CAD in our model.

Interestingly, it has been recently demonstrated that blood also contains circulating cell-free respiratory competent mitochondria, ${ }^{25}$ suggesting that the measure of mtDNAcn in the whole blood might better represent the health status. Moreover, despite the involvement of TMAO in mitochondrial metabolism has been presented, ${ }^{26-29}$ none of the previous investigations examined the association between blood mtDNAcn and TMA or TMAO levels in humans.

This study aims to investigate, in a population of 540 subjects of coronary artery disease (CAD) patients and controls, (a) if the mtDNA copy number measured in the whole blood is a marker of CAD; (b) if any association between mtDNAcn and TMAO or TMA levels exists; (c) if mtDNAcn is associated with other risk factors for CVD that are linked to metabolic alterations (ie, hypertension, ${ }^{30}$ diabetes,${ }^{31}$ glomerular filtration rate [GFR]) or environmental exposures (ie, smoking, $\left.{ }^{32} \mathrm{BMI}^{33}\right)$; (d) provide further insights on the effects of TMA and TMAO levels changes in CVD. The final goal is to identify relevant factors that can be used for CVD prognosis through adequate prediction models.

\section{2 | MATERIALS AND METHODS}

\section{1 | Study cohort recruitment and sample collection}

CAD patients were consecutively recruited in Wejherowo Cardiovascular Center with angiographically confirmed CAD or with angina referred to elective or urgent coronary angiography as inclusion criteria. To characterize the severity of CAD, all patients were classified into 1-, 2- or 3-vessel disease groups 
based on the presence of stenosis in major coronary arteries or their branches. CAD patients were further divided in subgroups based on severity of disease: mild severity (1 vessel involved) (MS) or high severity (two or three vessels involved) (HS). Moreover, CAD patients were divided into stable angina (SA), and acute coronary syndrome (ACS) patients. SA patients were treated with statins for a secondary prevention of cardiovascular morbidity. For this reason, despite dyslipidemia being an established risk factor for CAD, blood lipid measurements (Table S1) were excluded from the analysis. Control subjects without a self-reported medical history of CVD were recruited in the same region. The study was approved by the Regional Bioethical Committee (RBC) in Gdansk (KB-27/16 and KB 32-17) and registered at clinicaltrials.gov (NCT03899389). All methods were carried out in accordance with relevant guidelines and regulations approved by RBC. Informed consent was obtained from all subjects.

Venous blood samples were collected in EDTA-containing tubes. The plasma samples were prepared by centrifugation at $1300 \mathrm{~g}$ for $10 \mathrm{~min}$ at $18-25^{\circ} \mathrm{C}$, and were kept frozen at $-80^{\circ} \mathrm{C}$

\section{2 | Assessment of variables related to cardiovascular risk}

Plasma TMA and TMAO were determined by the UltraPerformance Liquid Chromatography (UHPLC) tandem mass spectrometry method, as previously described. ${ }^{34}$ Troponin, as an established biomarker of ACS, ${ }^{35,36}$ was determined in hospital diagnostic laboratory on Dimension EXL with LOCI Module Integrated Chemistry System (Siemens Healthcare $\mathrm{GmbH}$, Erlangen, Germany) using high sensitivity cardiac troponin I test (Dimension EXL hs-cTnI assay, Siemens Healthcare GmbH, Erlangen, Germany). ${ }^{37}$

Body mass index (BMI) was calculated as weight in kilograms divided by height in meters squared. Hypertension and diabetes were diagnosed by medical doctors in diagnostic processes and were reported by subjects, as well as smoking habits (present or past), in self-reported questionnaires. GFR was calculated by Cockcroft-Gault Equation. ${ }^{38}$

\section{3 | MtDNAcn assessment}

Genomic DNA was extracted from whole blood using the kit for genomic DNA purification (A\&A Biotechnology, Gdynia, Poland). All samples have been processed under the same conditions and with the same DNA extraction method because variation of the DNA extraction method might affect the evaluation of mtDNAcn. ${ }^{39}$ Relative mtDNAcn quantification $^{40}$ (considering nDNA as a normalizer), which is the current method of choice for mtDNAcn assessment, ${ }^{19}$ was performed by real-time PCR (Biorad CFX96). Briefly, the cycle threshold $\left(C_{\mathrm{t}}\right)$ values of a mitochondrial-specific and nuclearspecific target were determined in triplicate for each sample. The difference in $C_{\mathrm{t}}$ values $\left(\Delta C_{\mathrm{t}}\right)$ between the mitochondrial and nuclear gene for each sample is calculated and $2^{-\Delta C t}$ represents a relative measure of mtDNAcn. The following genes have been amplified for the detection of mitochondrial and nuclear DNA, respectively, using the listed primers: mtDNAtRNALeu (fw: 5'-CACCCAAGAACAGGGTTTGT-3'; rv: 5'-TGGCCATGGGTATGTTGTTA-3') for mitochondrial DNA, and beta-2-microglobulin (B2M) (fw: 5'-TGCTGTCTCCATGTTTGATGTATCT-3'; rv: 5'-TCTCTGCTCCCCACCTCTAAGT-3') for the nuclear DNA. These primers have been verified by Fazzini and colleagues $^{39}$ for their specificity (unique amplification of mtDNA) and for the absence of co-amplified nuclear insertions of mitochondrial origin (NUMTs). An inter-run calibrator sample was used to adjust the results obtained from different amplification plates. All samples were anonymized for laboratory personnel.

\subsection{Statistical analysis}

Power analysis for studying mtDNAcn in this population was performed according to the effect size reported by the metaanalysis from Yue and colleagues ${ }^{24}$ and revealed a power $>0.99$. The Shapiro-Wilk test was used for the analysis of the normality of data distribution. Spearman correlation or linear regression (adjusting for confounding variables) was used for testing the correlation among continuous variables. Bonferroni's correction was applied to confirm statistical significance in multiple correlations. Chi-square test, KruskalWallis test, and Generalized Linear Model (GLM) were used to test differences in the analyzed variables among groups adjusting for covariates. A stepwise logistic regression model was applied to identify (according to Wald statistics) which of the variables predicted the cardiovascular risk. PrecisionRecall (PR) curves were used to evaluate the performance of the prediction model when considering a database with unbalanced classes, while Receiver Operating Characteristic (ROC) curves were considered when classes were numerically balanced. For completeness of information, we reported both the models in the figures.

For testing the predictive biomarkers, the dataset has been divided into two parts: the training set and the testing set. The former contains $75 \%$ of the whole dataset while the latter contains the remaining $25 \%$. We used the training set for training the logistic classifier (discovery stage) and then the testing set to evaluate it by computing the PR curve (validation stage). Precision and recall have been calculated as follows:

$$
\text { Precision }=\mathrm{TP} /(\mathrm{TP}+\mathrm{FP})
$$




$$
\text { Recall }=\mathrm{TP} /(\mathrm{TP}+\mathrm{FN})
$$

where TP, True Positives; FN, False Negatives.

Technical replicates are described in each specific material and methods section and are not considered for the inference statistics (no inflation of units of analysis was performed). Two-sided $\mathrm{p}$ values have been calculated, and significant differences were attributed to $P<.05$.

\section{3 | RESULTS}

\section{1 | Descriptive statistics}

Five hundred and forty recruited subjects ( $65.7 \%$ male, $34.3 \%$ female) were analyzed in this study. Mean age of the population was $65( \pm 10)$ years old. Among the subjects, $46.7 \%$ $(\mathrm{n}=252)$ were smokers, $66.5 \%(\mathrm{n}=359)$ were diagnosed with hypertension, and $25.4 \%(\mathrm{n}=137)$ were diagnosed with diabetes. Among the recruited subjects, 151 were controls (28\%), 389 were CAD patients (72\%). Descriptive statistics for BMI, TMA, TMAO, TMAO/TMA ratio, and GFR in the total population are shown in Table 1. A detailed comparison of TMA, TMAO, BMI, and GFR in the CAD population vs controls in this population has been previously published ${ }^{34}$ and summarized in Table S2.

As expected, smoking (Pearson's chi-square $=4.86$; $P=.028$ ), hypertension (Pearson's chi-square $=60.79$; $P=6.3^{*} 10^{-15}$ ), and diabetes (Pearson's chi-square $=14.55$; $\left.P=1.3 * 10^{-4}\right)$ are confirmed as risk factors for CAD development (Figure S1). An increased BMI was also measured in CAD patients with respect to controls (controls: $27.8 \pm 4.1$; CAD: $28.8 \pm 4.5 ; P=.023$ ).

\section{2 | Association between mtDNAcn, CAD, and CVD risk factors}

In the studied population, blood mtDNAcn decreased with age (Spearman's Rho $=-0.101 ; P=.019$ ) (Figure S2). A significantly lower level of mtDNAcn was measured in the CAD group compared to controls (median values in controls vs CAD:

T A B L E 1 Descriptive statistics. Descriptive statistics on the whole population $(n=540)$ for the analyzed variables

\begin{tabular}{llrrrr} 
& n & \multicolumn{1}{c}{ Min } & \multicolumn{1}{c}{ Max } & Mean & \multicolumn{1}{c}{ SD } \\
BMI $\left(\mathrm{kg} / \mathrm{m}^{2}\right)$ & 540 & 18.00 & 45.00 & 28.50 & 4.40 \\
TMAO $(\mu \mathrm{M})$ & 540 & 0.01 & 37.50 & 5.37 & 4.50 \\
TMA $(\mu \mathrm{M})$ & 540 & 0.34 & 1.15 & 0.60 & 0.11 \\
TMAO/TMA & 540 & 0.01 & 64.87 & 8.86 & 7.35 \\
GFR $(\mathrm{mL} /$ & 540 & 9.71 & 289.96 & 88.17 & 33.76 \\
$\left.\min / 1.73 \mathrm{~m}^{2}\right)$ & & & & & \\
\hline
\end{tabular}

Abbreviation: SD, standard deviation
76.9 vs $51.5 ; P=2.6^{*} 10^{-12}$ ) (Figure 1 ). Analysis of covariates (age, sex, TMA, TMAO, GFR) revealed that this association is modulated by TMA $(P=.0001)$ and GFR $(P=.026)$.

Moreover, mtDNAcn was significantly lower in hypertensive than in normotensive individuals (median values, control $=59.8 ; \mathrm{CAD}=53.6 ; P=.002)($ Figure $2 \mathrm{~A})$. This association was modulated by TMA $(P=.0001)$ and GFR $(P=.002)$, but not by TMAO $(P=.549)$, sex $(P=.987)$, BMI $(P=.821)$ or age $(P=.231)$. However, it was no more significant when adjusting the analysis for CVD status $(P=.830)$, suggesting that $\mathrm{CAD}$ is the major driver of this association. Adjusting the analysis for age, sex, BMI, GFR, TMA and TMAO, the mtDNAcn did not differ in individuals diagnosed with diabetes $(P=.795)$ (Figure 2C), nor in smokers with respect to controls $(P=.082)$ (Figure 2B). However, the association with smoking was significant $(P=.006)$ when adjusting the analysis for $\mathrm{CAD}$, that contribute to explain the model $(P=.002)$, as well as GFR $(P=.016)$ and TMA $(P=.0001)$. MtDNAcn was directly correlated with GFR (Spearman's Rho $=0.103 ; P=.017$ ), suggesting that higher mtDNAcn can be associated with a better glomerular filtration capacity (Figure 2D). Adjusting the analysis for age, sex and BMI, the correlation is even stronger $(B=0.216 ; P=.001)$. It remains significant also after adjusting the analysis for CAD status $(B=0.155 ; P=.010)$. No significant direct association was measured between mtDNAcn and BMI in the whole population (Spearman's Rho $=0.044 ; P=.304$ ), neither adjusting the analysis for sex and age $(P=.310)$.

\section{3 | MtDNAcn correlates with TMA but not TMAO}

A direct correlation between mtDNAcn and TMA levels was measured (Spearman's Rho $=0.166 ; P=.0004$ ) (Figure 3A). Linear regression adjusting the analysis for age, sex, GFR, and BMI confirmed a strong association between TMA and mtDNAcn $\left(B=0.227 ; P=6.5^{*} 10^{-7}\right)$, with GFR $(B=0.206$; $P=.002)$ contributing to explain the mtDNAcn variance. This association remains significant even adjusting the analysis for the CAD status $\left(P=2.3^{*} 10^{-5}\right)$. No significant associations were detected between mtDNAcn and TMAO (Spearman's Rho $=0.020$; $P=.643$ ) (Figure 3B), neither adjusting the analysis for the previously mentioned variables $(P=.928)$. Similarly, no significant association was measured with TMAO/TMA (Spearman's Rho $=$ $-0.033 ; P=.441$ ) (Figure 3C), neither adjusting the analysis for confounding variables $(P=.598)$.

\section{4 | MtDNAcn, TMA, TMAO and troponin levels in CAD groups}

Troponin, a marker of acute myocardial injury, was higher in HS than MS $(P=.003)$ (Figure S3A) and in ACS than in SA 
F I G URE 1 Relative mtDNAcn in controls and CAD group

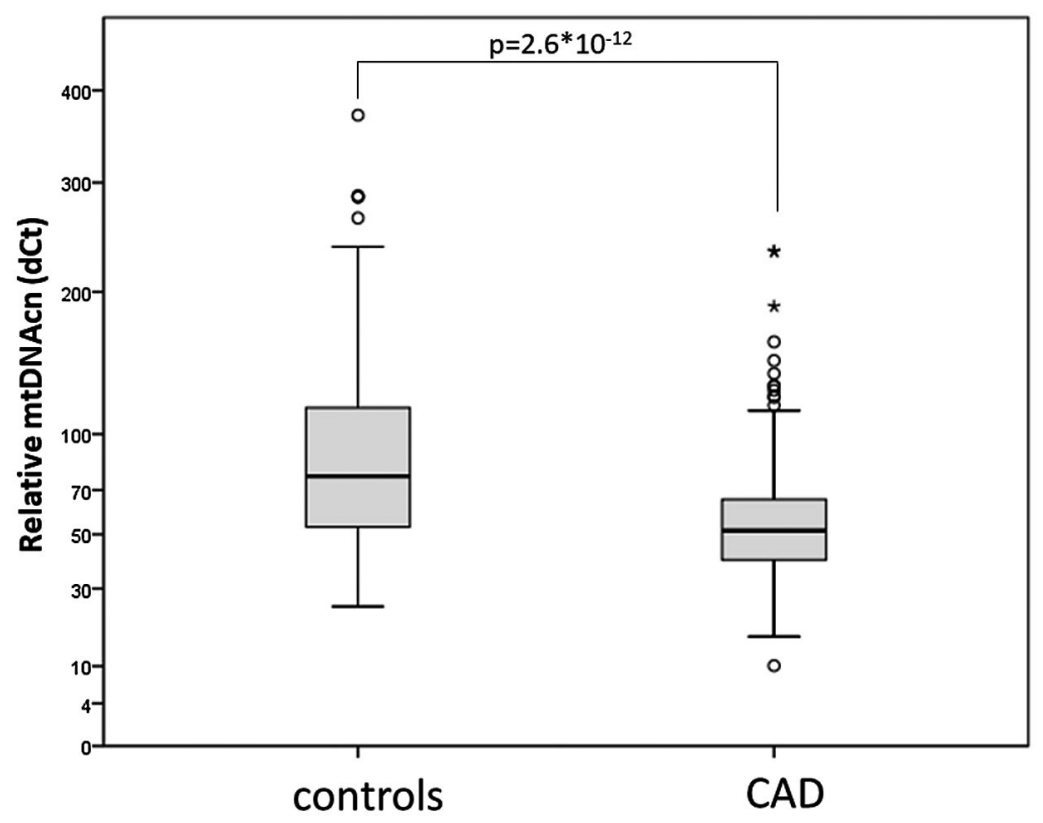

$\left(P=1.7 * 10^{-6}\right)$ (Figure S3B). On the other hand, mtDNAcn decreased with respect to controls in both MS $(P=.0001)$ and HS $(P=.0001)$, but did not significantly differ between MS and HS groups $(P=.666)$ (Figure S3C). Moreover, mtDNAcn was lower in both SA $(P=.0001)$ and ACS groups $(P=.0001)$ compared to controls, but no significant differences were measured between ACS and SC $(P=.186)$ for this biomarker (Figure S3D). Interestingly, a significant inverse correlation between mtDNAcn and troponin was measured in the CAD group (Spearman's Rho $=-0.218 ; P=1.5^{*} 10^{-5}$ ) (Figure S4). This association was confirmed also after adjusting the analysis for sex and age $(B=-0.451 \pm 0.218$; $P=.039)$ and can be considered reliable among acute cases (that are those that display higher troponin levels). No significant correlations were detected between troponin and TMA $(P=.554)$, TMAO $(P=.946)$, or TMA/TMAO $(P=.985)$ in the CAD group.

\section{5 | Correlations between TMA, TMAO, GFR, and mtDNAcn in controls and CAD patients}

To clarify the role of TMA in this complex picture, an exploratory analysis was performed to evaluate the correlations between TMA and TMAO in the control and CAD groups separately. Results showed that high TMA levels were not accompanied by increased TMAO in controls (Spearman's Rho $=0.065 ; P=.429$ ), but high TMA levels were associated with high TMAO levels in CAD patients (Spearman's Rho $=0.338$; $P=7.2 * 10^{-5}$ ) (Figure 4A). This correlation is even more significant adjusting the analysis for sex, age, BMI and GFR ( $B=0.246 ; P=1.4^{*} 10^{-6}$ ) in the CAD group, but not in the control group $(P=.964)$. This evidence corroborates the hypothesis that TMA levels are not per se linked to high TMAO.

Higher TMA levels are positively correlated to GFR in controls (Spearman's Rho $=0.257 ; P=.001$ ), while TMA is negatively associated to GFR levels in the CAD group (Spearman's Rho $=-0.260 ; P=2.1 * 10^{-7}$ ) (Figure 4B). By adjusting the analysis for sex, age and BMI, only the inverse correlation between GFR and TMA in the CAD group was confirmed $\left(B=-0.454 ; P=7.8^{*} 10^{-7}\right.$ ) after Bonferroni correction. This suggests a different metabolism of TMA and TMAO in the presence of cardiovascular disease, with respect to healthy conditions. Moreover, increased TMA levels correlated to higher mtDNAcn only in the controls (Spearman's Rho $=0.280, P=7.8^{*} 10^{-5}$ ), and not in the CAD group (Spearman's Rho $=0.076, P=.133$ ). However, this correlation between TMA and mtDNAcn in the controls was not significant when adjusting the analysis for sex, age and GFR $(P=.707)$.

By observing the TMAO/TMA ratio in the population divided by the cardiovascular health status in respect to $\mathrm{mtD}$ NAcn and GFR, we found that the TMAO/TMA ratio shows a significant inverse correlation with GFR in the CAD group (Spearman's Rho $=-0.307 ; P=5.8 * 10^{-6}$ ), which, on the other hand, is not significant in controls (Spearman's Rho = $-0.176 ; P=.180$ ) after Bonferroni correction (Figure 4C). This inverse correlation in the CAD group was confirmed also adjusting the analysis for sex and age $(B=-1.032$; $P=.0001)$. Additionally, the TMAO/TMA ratio was inversely correlated to mtDNAcn in the control group (Spearman's Rho $=-0.161 ; P=.049$ ), but not in the CAD group (Spearman's Rho $=-0.019 ; P=.712$ ). However, this correlation was not significant when adjusting the analysis for sex, age and GFR $(P=.292)$. 

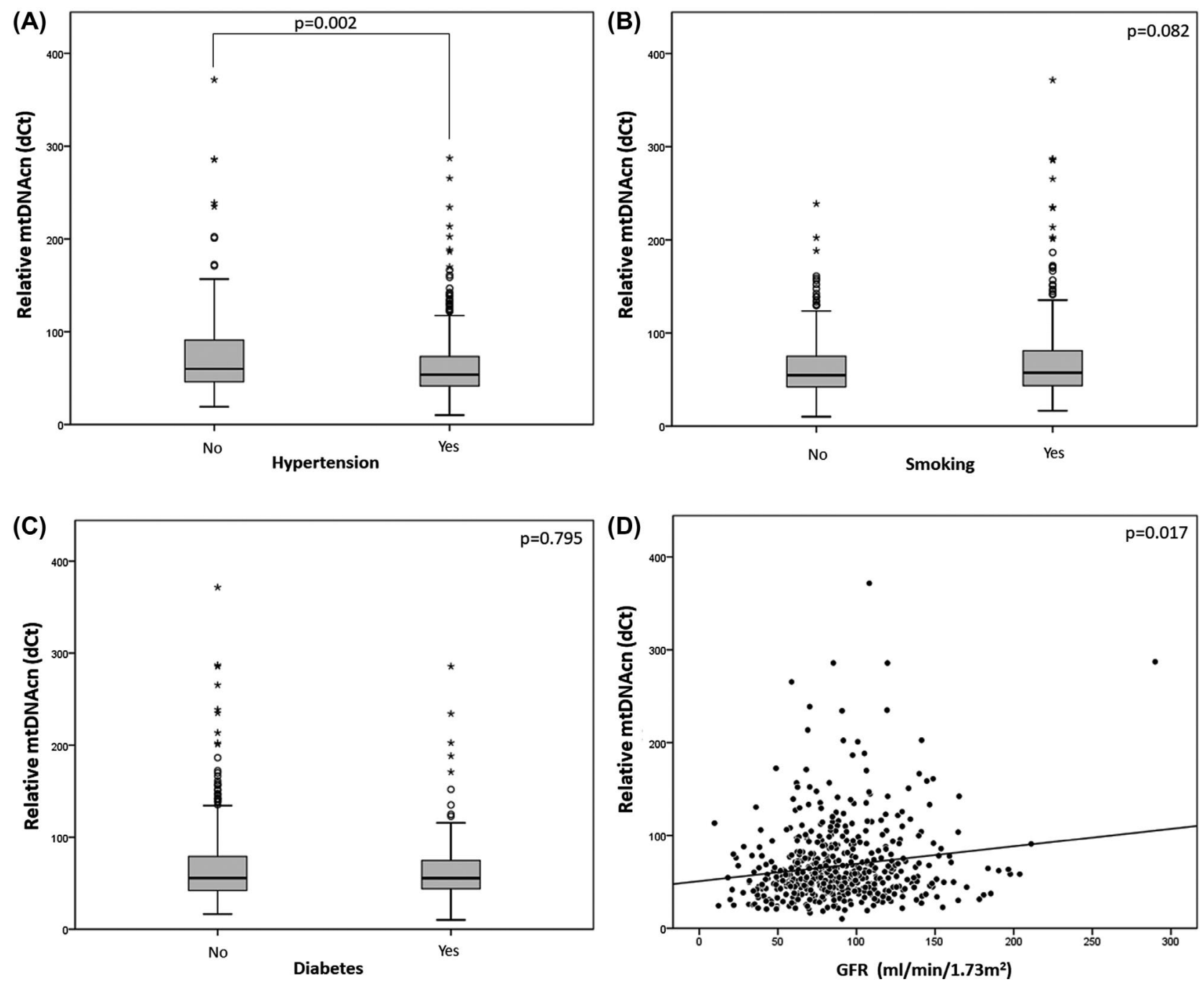

F I G U R E 2 Relative mtDNAcn and CAD risk factors exposures in the analyzed population: hypertension (A), smoking (B), diabetes (C), GFR (D). Row $P$ values are shown in the figures. Adjusted analysis and covariates are described in the main text

\section{6 | Identification of CAD prediction models and comparison with troponin}

A stepwise logistic regression analysis was applied to identify significant predictors of CAD. The following variables, all potential risk factors for CAD, were tested: age, sex, $\mathrm{mtD}$ NAcn, TMA, TMAO, diagnosis of hypertension and diabetes, smoking habits, GFR. A forward selection criterion was applied and Wald statistics was used to identify significant predictors. The variables that emerged as significant predictors of CAD are the following: mtDNAcn $(\mathrm{OR}=0.972 ; 95 \% \mathrm{CI}=$ [0.965-0.979]; $\left.P=7.6^{*} 10^{-9}\right)$; hypertension $(\mathrm{OR}=4.131 ; 95 \%$ $\left.\mathrm{CI}=[2.582-6.609] ; P=6.3 * 10^{-6}\right)$; sex $(\mathrm{OR}=0.574 ; 95 \% \mathrm{CI}$ $=[0.355-0.929] ; P=.024) ;$ smoking $(\mathrm{OR}=1.754 ; 95 \% \mathrm{CI}$ $=[1.083-2.841] ; P=.022)$; diabetes $(\mathrm{OR}=1.861 ; 95 \% \mathrm{CI}$ $=[1.011-3.425] ; P=.045)$. This 5 -step model correctly categorized $81.7 \%$ of cases. Good performances were obtained also by a 3-step model (including mtDNAcn, hypertension, and sex), that successfully categorized $80.3 \%$ of cases. TMAO and TMA are not significant CAD predictors according to this model. On the contrary, mtDNAcn is the first element to enter in the stepwise forward analysis, thus affecting the goodness of the prediction and the information carried by the other variables. This evidence suggests that mtDNAcn might significantly improve the population risk stratification for CAD, in addition to other known risk factors.

To confirm this hypothesis, the PR curves were calculated (Figure 5) to evaluate the performance of both prediction models previously identified by the stepwise forward logistic regression. PR curve is used to evaluate the performance of a classifier (to compare classifiers, too) for unbalanced classes through the computation of the area under the curve (AUC). This value is a number between 0 and 1: the bigger the value, the better the model. PR curve, as the name suggests, is obtained using precision and recall, two evaluation metrics that are able to deal with unbalanced classes ${ }^{41}$ as in this case. 

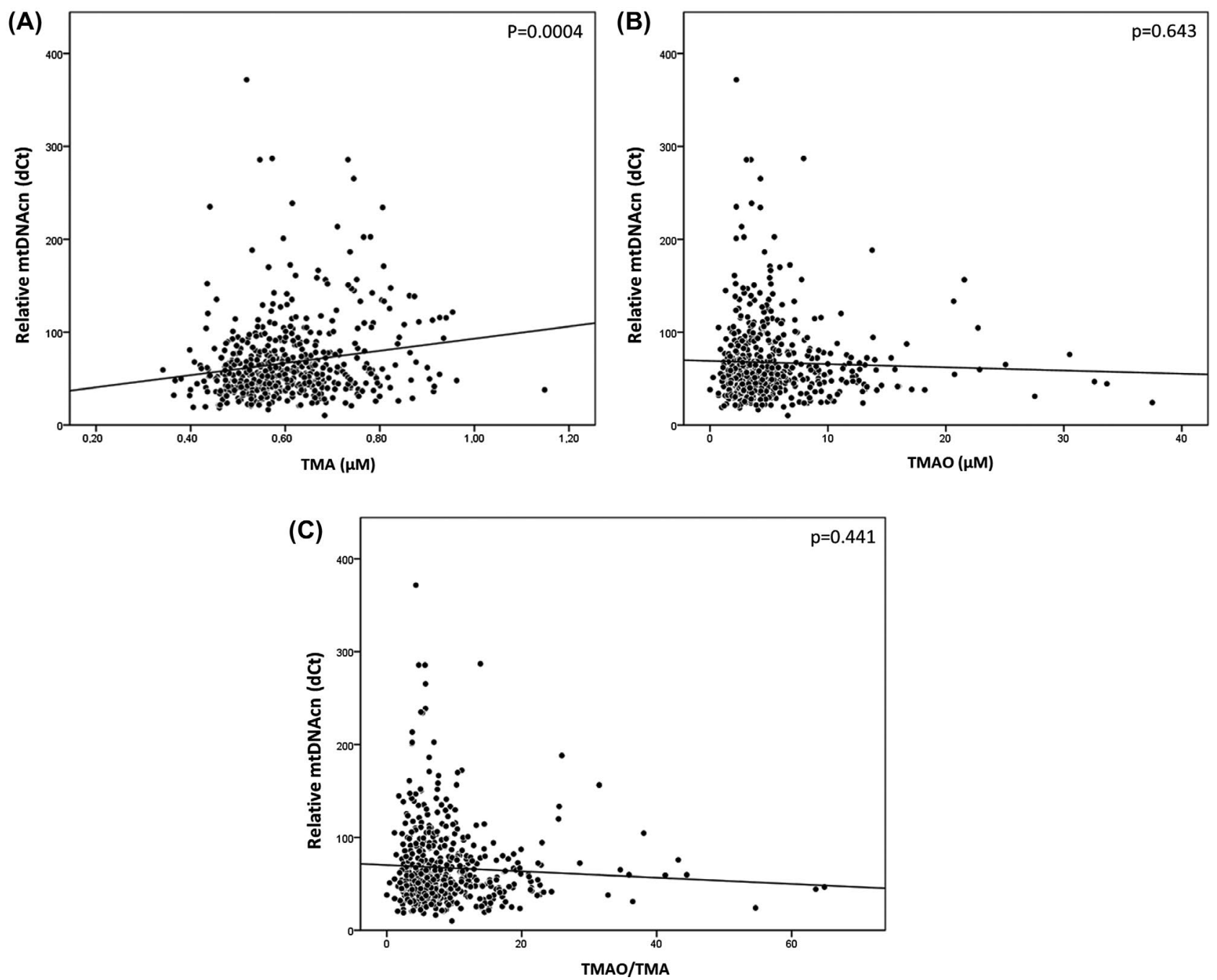

F I G U R E 3 Correlations between mtDNAcn and TMA (A), TMAO (B) or TMAO/TMA (C) in the analyzed population. TMA levels correlates with mtDNAcn $(P<.0001$, adjusted for age and sex $)$. The association remains significant also correcting the analysis for GFR as well $(P<.0001)$. No significant correlations were detected between TMAO and mtDNA or TMA/TMAO ratio and mtDNAcn

PR shows an AUC equal to 0.894 for the 3-step model, with mtDNAcn, hypertension and sex applied as predictors. For the 5-step model (with mtDNAcn, hypertension, sex, smoking, and diabetes applied as predictors), the resulting AUC was 0.901 . Figure 5 shows the PR curves and the confusion matrices for both models, concluding that their performances are good and similar.

In addition, we tested the ability of these two models to distinguish between acute or stable CAD patients (Figure 6), or between different degrees of CAD severity (Figure 7), and we compared them to the performance of troponin. Results confirmed that troponin can distinguish between stable and acute cases (ROC AUC $=0.79$ ), while none of the new proposed biomarkers (3-steps, ROC AUC $=0.58 ; 5$-steps, ROC AUC $=0.53$ ) were able to confidently predict the acute or stable status of the disease (Figure 6D-F respectively). Remarkably, our 5-step $(\mathrm{PR}$ AUC $=0.703)$, and 3-step model $(\mathrm{PR}$ AUC $=0.778)$ showed to be better predictors of severity than troponin (PR AUC $=0.667$ ). (Figure 7A-C respectively). Therefore, the proposed 3-step model, including mtDNAcn, sex, and hypertension, was confirmed as an interesting predictor of cardiovascular health in this population.

\section{4 | DISCUSSION}

Alterations in TMAO metabolism ${ }^{42}$ and mitochondrial dynamics ${ }^{43}$ have been previously independently identified as risk factors for cardiovascular disease development. We have found that mtDNA copy number, measured in the whole blood, is lower in CAD patients than in controls. This is coherent with previous investigations reviewed by Yue et al, ${ }^{24}$ although the reported studies had determined mtDNAcn in buffy coat/circulating leukocytes. Recently, it has been demonstrated that blood contains circulating cell-free respiratory 

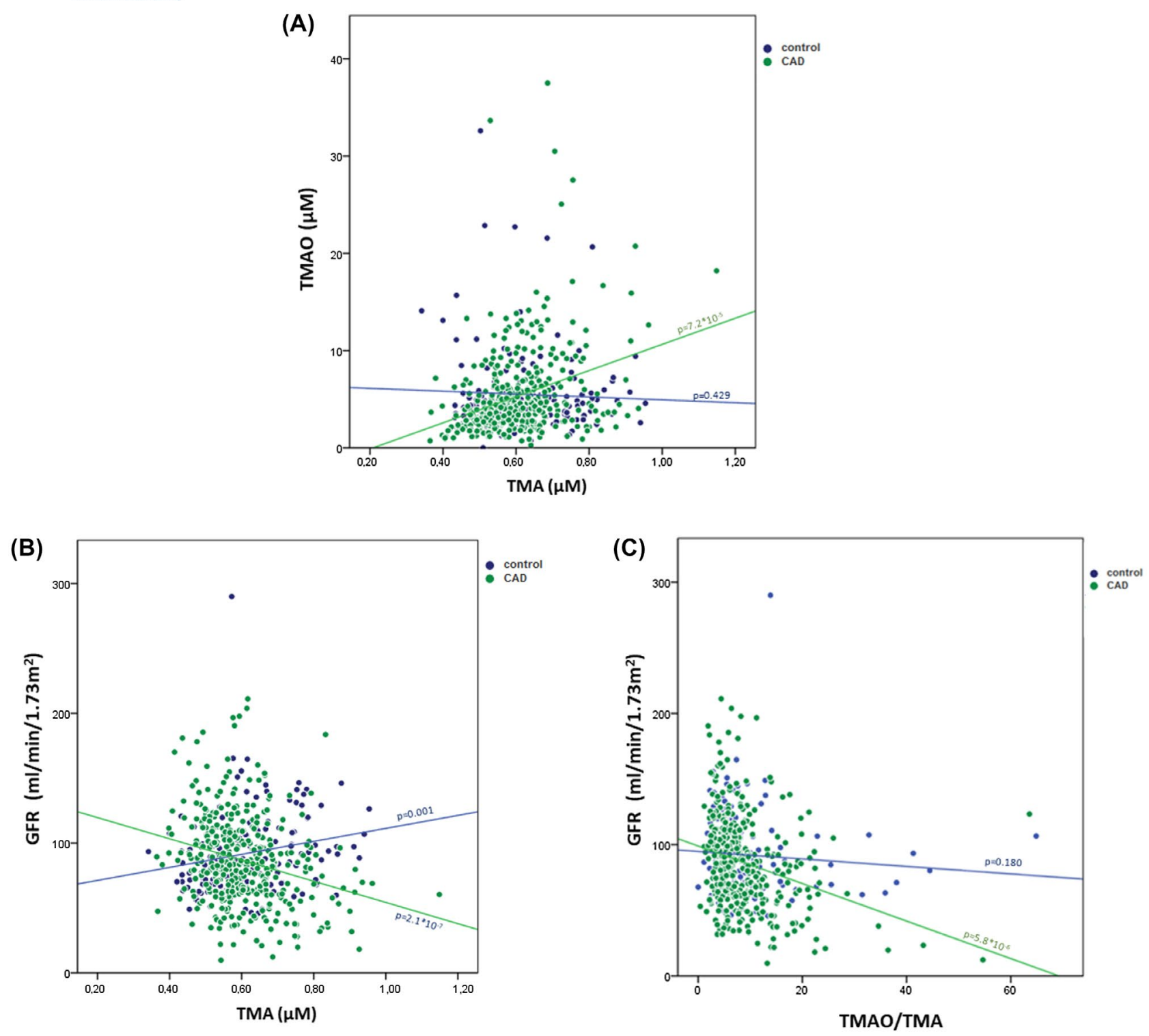

F I G URE 4 Correlations between TMA and TMAO (A), TMA and GFR (B), TMAO/TMA ratio and GFR (C) in the population divided for groups (controls vs CAD)

competent mitochondria, ${ }^{25}$ and a reduction of mtDNAcn was also detected in the whole blood of peripheral arterial disease male patients compared to controls. ${ }^{44}$ Therefore, our findings about the reduction of mtDNAcn in the whole blood of CAD patients support the interest towards mtDNAcn measurement as an easy-accessible biomarker for cardiovascular disease risk stratification.

To explain the observed decrease of mtDNAcn in CAD patients, we tested the correlation of this biomarker with other established risk factors for CVD. In our population, mtDNAcn decreased with age, according to previous evidence examining mtDNAcn in blood cells. ${ }^{45}$ In addition, we found a correlation between mtDNAcn, hypertension and GFR, which are established risk factors for CVD. ${ }^{46-48}$ In particular, mtDNAcn was decreased in hypertensive subjects. Even if the presence of CAD is a confounder for this association in our population, previous hypothesis linked mtDNAcn and hypertension, ${ }^{49}$ which is also interesting considering the role of mitochondrial oxidative stress in CAD. ${ }^{50}$ Nevertheless, only a few data on this topic have been collected until now, and a clear mechanistic explanation is missing. Lei et $\mathrm{al}^{51}$ measured $\mathrm{mtD}$ NAcn in white blood cells of a coal mining group located in northern China, and reported no differences between hypertensive and healthy controls. An elevated urinary mtDNAcn was detected in hypertensive patients, and it was correlated with markers of renal injury and dysfunction. ${ }^{52,53}$ The authors explained this observation considering that fragments of the mitochondrial genome released from dying cells might be considered surrogate markers of mitochondrial injury. Prestes et $\mathrm{al}^{49}$ also suggested that alterations of mtDNAcn in a cell can impair mitochondrial 

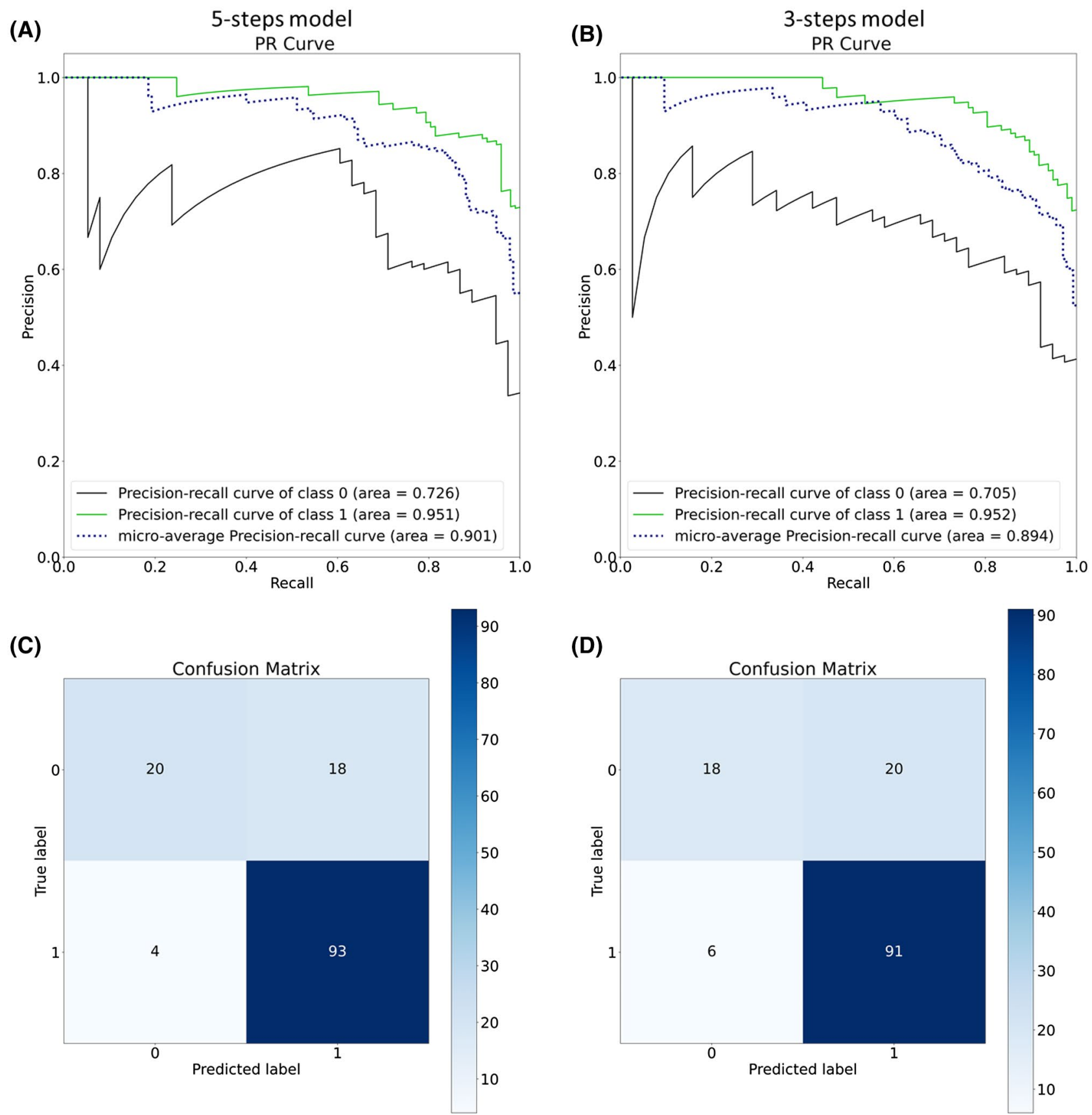

(D)

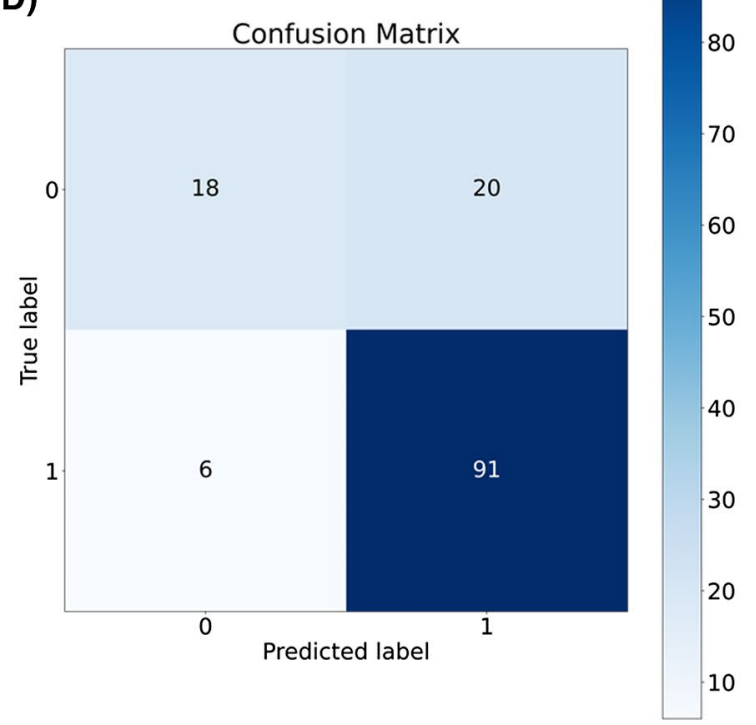

F I G U R E 5 PR curves for CAD prediction with 3 features (A, 5-step model: including mtDNAcn, hypertension, sex) or 5 features (B, 3 -step model: including mtDNAcn, hypertension, sex, smoking, diabetes). Panel C and D show the confusion matrices for the two models (5-step and 3step model, respectively). 5-step model, Accuracy: 0.837; Sensitivity: 0.526; Specificity: 0.959. 3-step model, Accuracy: 0.807; Sensitivity: 0.474; Specificity: 0.938

respiration and a reduction of mtDNAcn might represent a surrogate measure for reduced mitochondrial function, which is coherent with what we observed in our CAD group. In addition, elevation of mtDNAcn in urine samples has been shown in progressive acute kidney injury patients ${ }^{54}$ and this parameter has been associated with glomerular hyperfiltration in obese African American hypertensive patients. ${ }^{53}$ All these data support the involvement of mitochondrial dynamics in both the alteration of kidney functions and hypertension. In our population, mtDNAcn decreased proportionally with GFR, in accordance with previous evidence, suggesting that a higher blood mtDNA copy number is associated with a lower risk of incident chronic kidney disease. ${ }^{55}$ The reduction of mtDNAcn in individuals with lower GFR might be explained by the mitochondrial dysfunctions in kidney cells implicated in the pathogenesis of chronic kidney diseases, ${ }^{56-58}$ and by the exposure to reactive oxygen species that can damage 
(A)

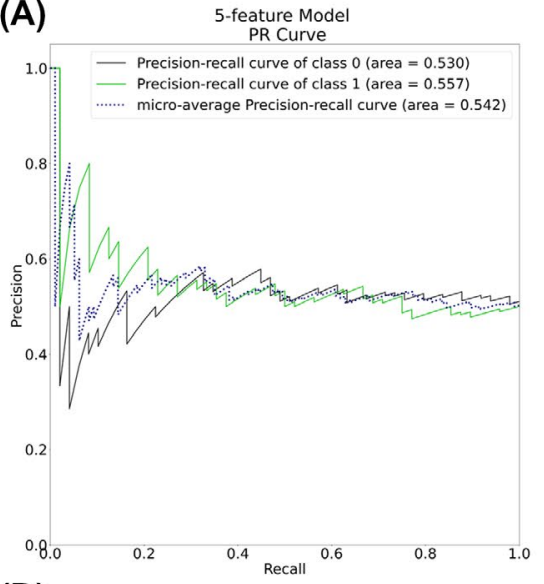

(D)

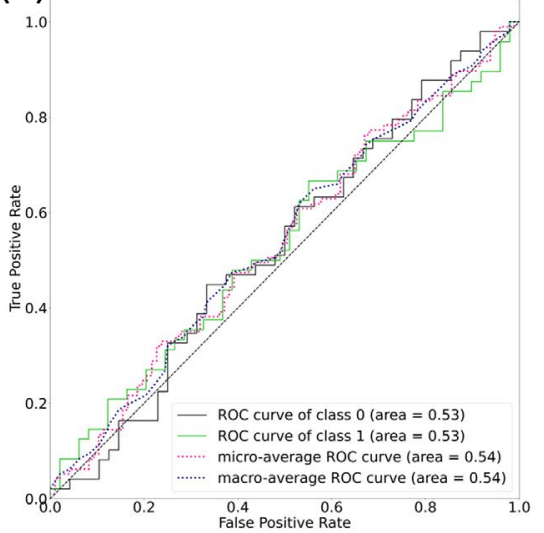

(G)

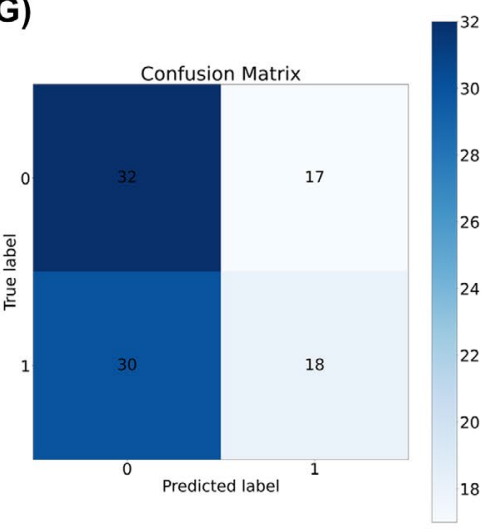

(B)

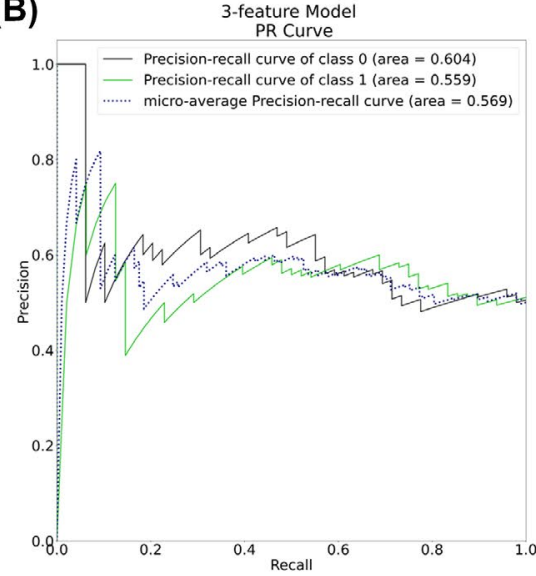

(E)

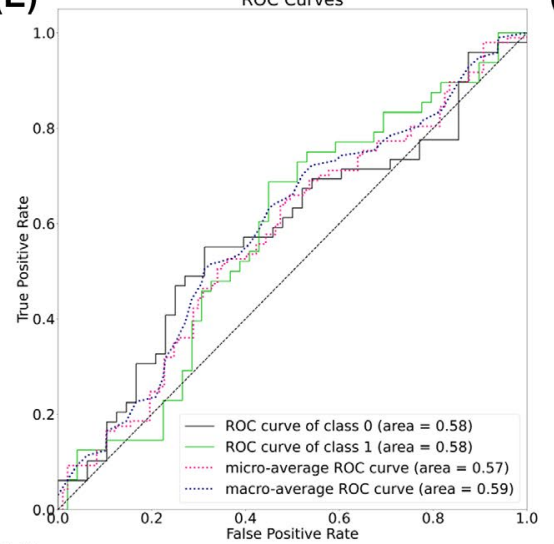

(H)

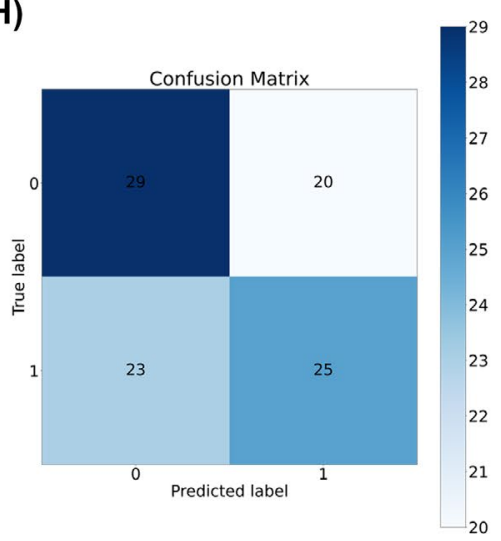

(C)

Troponin Model

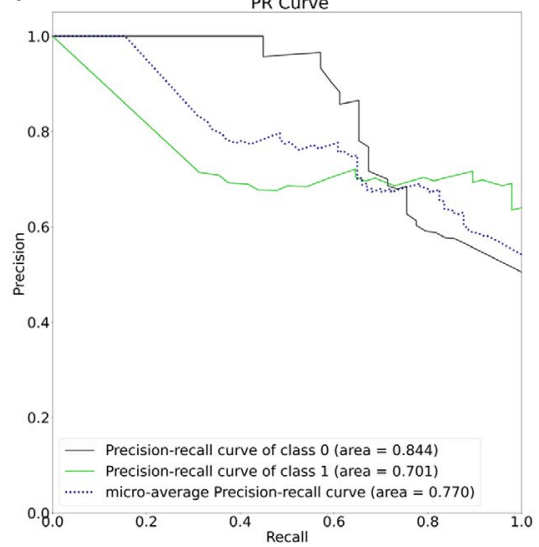

(F)

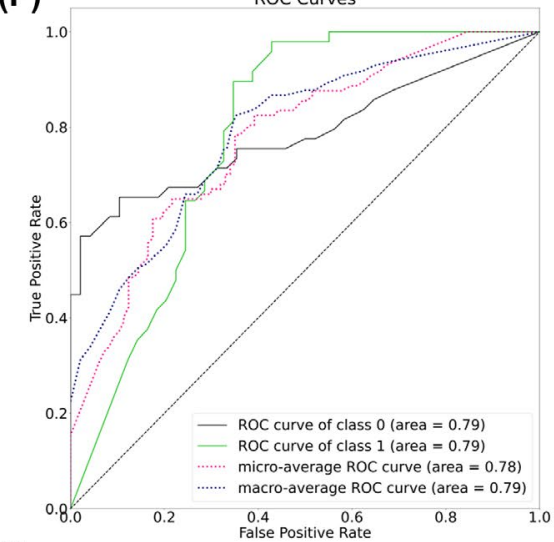

(I)

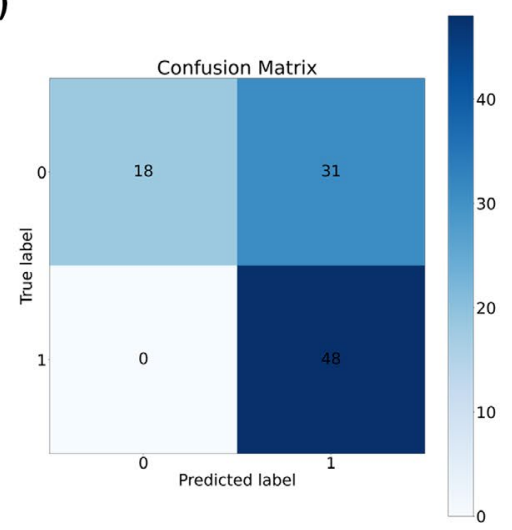

F IG U RE 6 PR curves, ROC curves and confusion matrices for prediction of the disease state (SA vs ACS) in the CAD group. A, D, G panels are referred to the 5-step model; B, E, H panels are referred to the 3-step model; C, F, I panels are referred to the troponin. 5-steps model, Accuracy: 0.515; Sensitivity: 0.653; Specificity: 0.375; 3-step model, Accuracy: 0.557, Sensitivity: 0.592, Specificity: 0.521; troponin, Accuracy: 0.681 , Sensitivity: 0.367 , Specificity: 1.0

the mitochondrial DNA replication enzyme (polymerase $\gamma$ ), leading to a reduction in mtDNA copy number. ${ }^{59}$ On the other hand, diabetes, that has been previously related to both the increase and decrease of mtDNAcn, ${ }^{60,61}$ was not directly associated with mtDNAcn in our study. This evidence cannot definitely exclude an influence of diabetes on mtDNAcn levels, but the findings are still inconclusive to demonstrate a direct association between these two variables. Recently, a meta-analysis has shown that metabolic parameters (waist circumference, BMI and triglycerides) are major mediators of this association. ${ }^{62}$ Nevertheless, the diagnosis of diabetes is still informative in this context, as it was one of the significant predictors of CAD in our precision-recall analysis if considered together with mtDNAcn, sex, hypertension, and smoking. Dyslipidemia, which is also an established risk factor for CVD, was not considered in this study because CAD patients were treated with statins for secondary prevention 
(A)

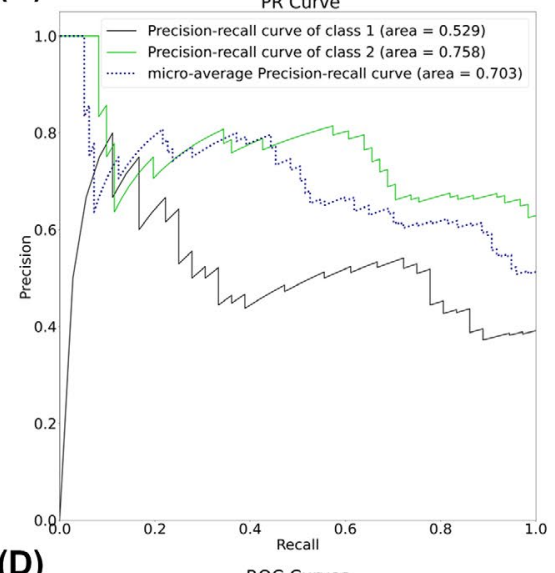

(D)
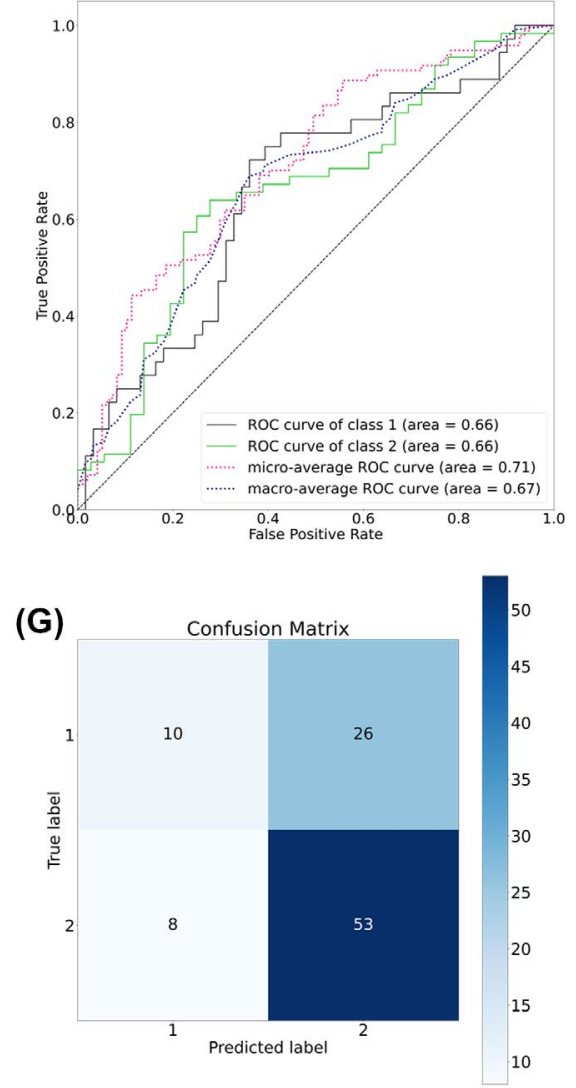

(B)

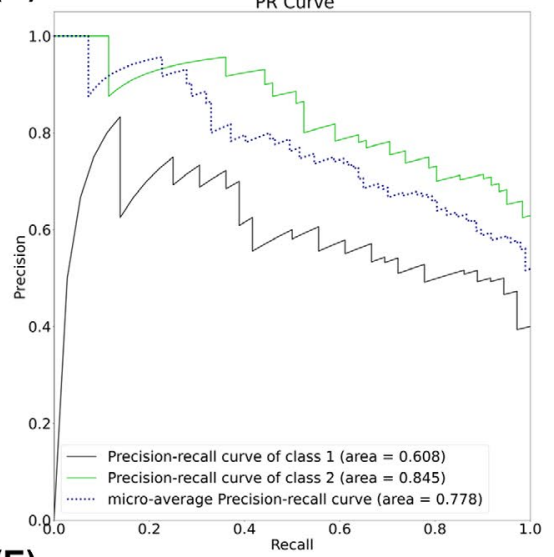

(E)

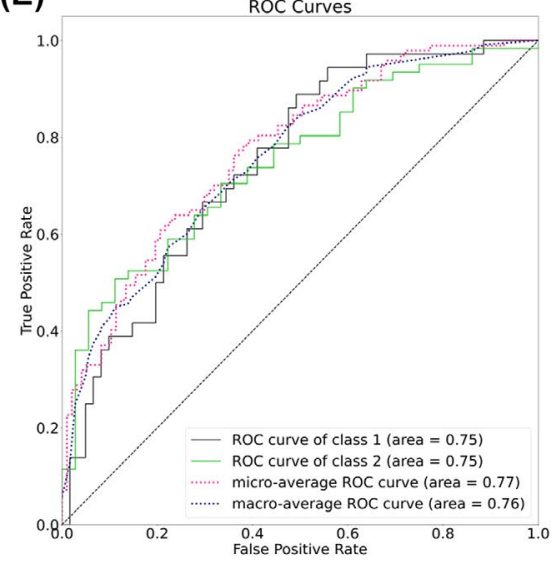

(C) Troponin Model

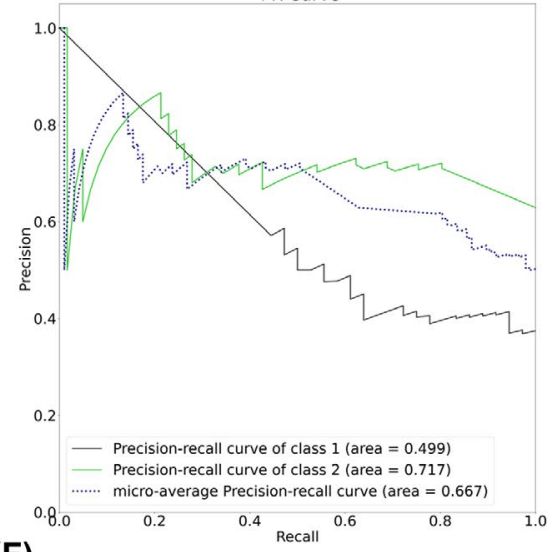

(F)

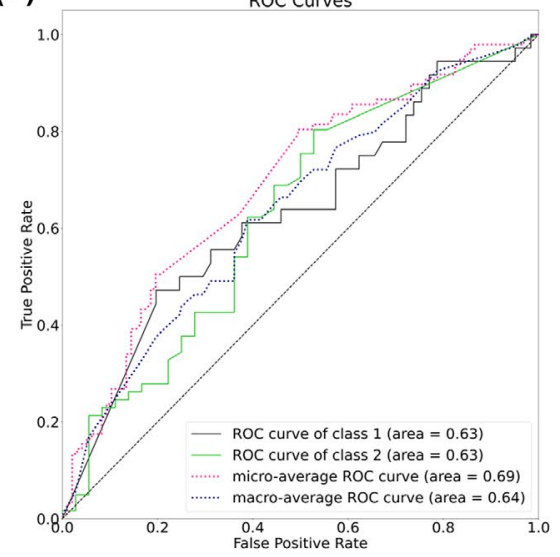

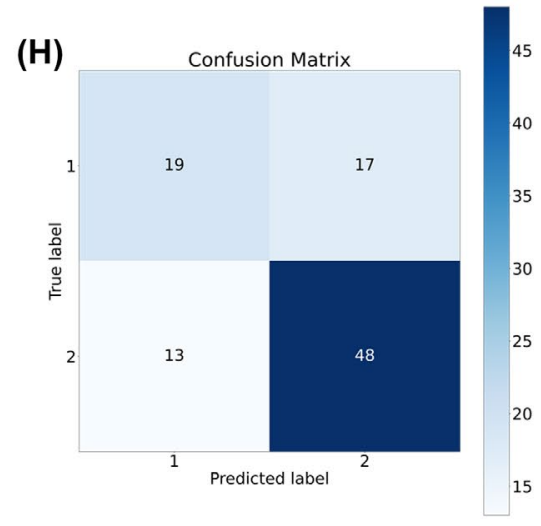

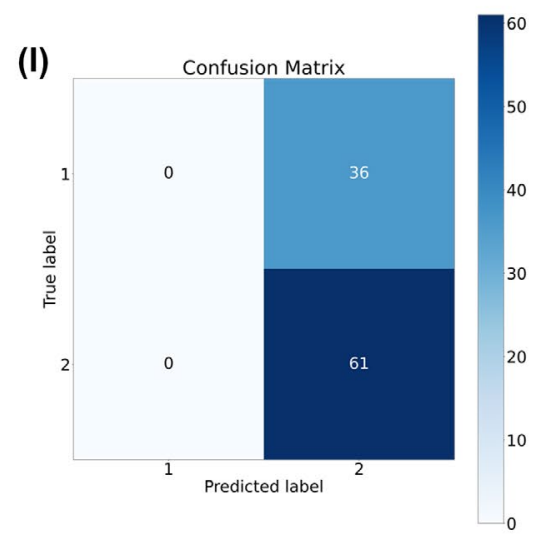

F IG U RE 7 PR curves, ROC curves and confusion matrices for prediction of disease severity in the CAD group. A, D, G panels are referred to the 5-step model; B, E, H panels are referred to the 3-step model; C, F, I panels are referred to the troponin. 5-step model, Accuracy: 0.650; Sensitivity: 0.279; Specificity: 0.869. 3-steps model, Accuracy: 0.691, Sensitivity: 0.528, Specificity: 0.787. Troponin, Accuracy: 0.628, Sensitivity: 0.0 , Specificity: 1.0

of cardiovascular events. Thus, data on blood lipids were not considered reliable in this population. Interestingly, mtDNAcn showed an inverse correlation with troponin levels, corroborating the hypothesis that lower mtDNAcn is predictive of worse cardiovascular health. Troponin was confirmed as a good biomarker of CAD in the acute phase. On the contrary, mtDNAcn increases both in acute and stable conditions and was predictive of disease severity when considered with sex and hypertension diagnosis.
This is in accordance with recent evidence suggesting that mtDNAcn is linked to cardiovascular disease patient phenotypes. ${ }^{63}$

In addition to classical risk factors for CVD, for the first time, we also tested correlations between mtDNAcn and TMAO metabolism. While no associations with TMAO were measured, mtDNAcn correlated with TMA levels in the whole population. This finding suggests that a reduction of mitochondrial functions might occur in individuals 
that display lower TMA. This is in accordance with our previously published data describing a subtle but significant reduction of TMA in CAD patients with respect to controls, ${ }^{34}$ while no significant differences were measured for TMAO between the two groups. A definite explanation of this phenomenon is still to be identified. In our cohort, a direct correlation between TMA and TMAO can be measured only in the CAD group, while we did not observe a proportional increase of TMAO in controls with higher TMA levels. Preliminary evidence suggested that TMA might have a role per se in CVD. ${ }^{64-66}$ Jaworska et al showed that TMA was inversely correlated with the estimated glomerular filtration rate in humans; moreover, TMA reduced cardiomyocyte viability in vitro, while TMAO was even protective against TMA-induced cytotoxicity. ${ }^{64}$ The authors hypothesized a detrimental effect of TMA due to its role in triggering the degradation of protein structures (ie, albumin). The possibility of stable protein-TMA complexes (especially in the condition of increased oxidative stress, as occurring in inflammatory conditions ${ }^{67-69}$ ), together with the increased ratio of conversion of TMA into TMAO might provide a possible explanation for the low TMA levels measured in CAD patients and suggest a different fate and metabolism of TMA in CVD respect to healthy people. Studies further investigating this hypothesis are ongoing in our laboratories.

A limitation of this study is the absence of data on urinary TMA and TMAO excretion. Moreover, although a positive correlation between the number of mitochondria and mtDNAcn has been demonstrated ${ }^{70}$ mtDNAcn is not always a reliable predictor for mitochondrial abundance, ${ }^{71-74}$ probably because of the existence of compensatory effects. Thus, our study supports a potential involvement of TMA (but not TMAO) in CVD pathogenesis; nonetheless, further mechanistic investigations are necessary to clarify the real role of this metabolite in CVD and its usage as a clinical biomarker.

In this regard, we tested the possibility to predict CAD by using all measured variables, including mtDNAcn and other classical CVD risk factors. A logistic regression model identified decreased mtDNAcn, male gender, diagnosis of hypertension, smoke habits, and diagnosis of diabetes as significant risk factors for CAD development in this population; conversely, neither TMA nor TMAO have been included as significant predictors. The precision-recall analysis showed that it is possible to have a good prediction of CAD by including information limited to mtDNAcn, sex, and hypertension diagnosis. Indeed, our 3-step prediction model (including mtDNAcn, sex, and hypertensions) displayed the best performance in distinguishing not only healthy subjects from CAD patients (even in stable conditions and with a very high true negative rate), but also patients with different degrees of disease. This confirms the hypothesis that mtDNAcn (considered together with other risk factors for CVD) could be an informative parameter describing cardiovascular health. Thus, validation in other cohorts of these 3 easy-to-measure parameters could promote their usage in the risk stratification for CVD at population level.

In conclusion, although we cannot infer any causal direction of the relationship between mtDNAcn and CAD, we consider unlikely that mtDNAcn has a direct role in increasing the risk for cardiovascular events (despite some animal studies have detected mtDNA damage before arterial wall lesions occurred ${ }^{75,76}$ ). The precise mechanism underlying the association between mtDNA damage and atherosclerosis is still poorly understood. However, it is well-known that mtDNA is a major target of oxidative stressors ${ }^{22}$ and mitochondrial dysfunctions promote the development of pathological conditions; on the other hand, caloric restriction and physical activity have been shown to limit mtDNA damage. $^{77,78}$ Indeed, this biomarker appears to vary in accordance with several risk factors for CVD. Thus, mtDNAcn reduction might represent an alarm signal for impaired mitochondrial homeostasis. Of note, cardiometabolic risk factors such as high blood pressure, obesity, and dyslipidemia have been previously shown to modify mtDNAcn. ${ }^{79,80}$ Thus, the hypothesis that environment and lifestyle factors might have major effects on mtDNAcn has been raised. Several pollutants (eg, benzene, ${ }^{81}$ particulate matter, ${ }^{82}$ and polycyclic aromatic hydrocarbons ${ }^{83}$ ) have been associated with changes in mtDNAcn. In addition, dietary factors, ${ }^{84,85}$ especially salt intake, ${ }^{86}$ might modify the mtDNAcn in blood. This is particularly interesting in the context of CVD prevention. Thus, mtDNAcn appears as a plastic biomarker that might change in response to environmental and dietetic factors. Further research on modifiable CVD risk factors influencing mtDNA copy number may improve the prevention and treatment of this multifactorial pathology, making mtDNAcn a new tool to monitor complex disorders related to both metabolic imbalances and environmental insults at the molecular level. ${ }^{21}$

\section{CONFLICT OF INTERESTS}

The authors declare no competing interests.

\section{AUTHOR CONTRIBUTIONS}

Concept and design: L. Bordoni, R. Gabbianelli, R.A. Olek; Study cohort recruitment: L. Lewicki, J.J. Samulak; sample processing: I. Petracci, I. Pelikant-Malecka, A. Radulska; Statistical analysis: L. Bordoni and M. Piangerelli; Drafting of the manuscript and data analysis: L. Bordoni; Critical revision of the manuscript: R.A. Olek, R. Gabbianelli; Supervision: R. Gabbianelli, L. Lewicki, R.A. Olek. All the authors approved the final version of the manuscript. 


\section{ORCID}

Laura Bordoni (D) https://orcid.org/0000-0001-6968-1164

Marco Piangerelli (D) https://orcid.

org/0000-0002-8545-3740

Rosita Gabbianelli (iD https://orcid.

org/0000-0003-0037-7740

Robert A. Olek (D) https://orcid.org/0000-0002-3714-7386

\section{REFERENCES}

1. Roth GA, Johnson C, Abajobir A, et al. Global, regional, and national burden of cardiovascular diseases for 10 causes, 1990 to 2015. J Am Coll Cardiol. 2017;70:1-25.

2. Wang J, Tan G-J, Han L-N, Bai Y-Y, He M, Liu H-B. Novel biomarkers for cardiovascular risk prediction. $J$ Geriatr Cardiol. 2017; 14:135-150.

3. Wang Z, Klipfell E, Bennett BJ, et al. Gut flora metabolism of phosphatidylcholine promotes cardiovascular disease. Nature. 2011;472:57-63.

4. Tang WHW, Wang Z, Levison BS, et al. Intestinal microbial metabolism of phosphatidylcholine and cardiovascular risk. $N$ Engl J Med. 2013;368:1575-1584.

5. Koeth RA, Wang Z, Levison BS, et al. Intestinal microbiota metabolism of L-carnitine, a nutrient in red meat, promotes atherosclerosis. Nat Med. 2013;19:576-585.

6. Senthong V, Wang Z, Li XS, et al. Intestinal microbiota-generated metabolite trimethylamine-N-oxide and 5-year mortality risk in stable coronary artery disease: the contributory role of intestinal microbiota in a COURAGE-like patient cohort. J Am Heart Assoc. 2016;5:e002816.

7. Kanitsoraphan C, Rattanawong P, Charoensri S, Senthong V. Trimethylamine N-oxide and risk of cardiovascular disease and mortality. Curr Nutr Rep. 2018;7:207-213.

8. Zhu W, Gregory JC, Org E, et al. Gut microbial metabolite TMAO enhances platelet hyperreactivity and thrombosis risk. Cell. 2016;165:111-124.

9. Seldin MM, Meng Y, Qi H, et al. Trimethylamine N-oxide promotes vascular inflammation through signaling of mitogenactivated protein kinase and nuclear factor- $\mathrm{\kappa B} . J$ Am Heart Assoc. 2016;5:e002767.

10. Haghikia A, Li XS, Liman TG, et al. Gut microbiota-dependent trimethylamine $\mathrm{N}$-oxide predicts risk of cardiovascular events in patients with stroke and is related to proinflammatory monocytes. Arterioscler Thromb Vasc Biol. 2018;38:2225-2235.

11. Olek RA, Samulak JJ, Sawicka AK, et al. Increased trimethylamine $\mathrm{N}$-oxide is not associated with oxidative stress markers in healthy aged women. Oxid Med Cell Longev. 2019;2019:1-6.

12. Samulak JJ, Sawicka AK, Hartmane D, et al. L-carnitine supplementation increases trimethylamine-N-oxide but not markers of atherosclerosis in healthy aged women. Ann Nutr Metab. 2019;74:11-17.

13. Bordoni L, Sawicka AK, Szarmach A, Winklewski PJ, Olek RA, Gabbianelli R. A pilot study on the effects of 1-carnitine and trimethylamine-N-oxide on platelet mitochondrial DNA methylation and CVD biomarkers in aged women. Int J Mol Sci. 2020;21(3):1047.

14. Jia Y, Shuo-Xi L, Yan H, et al. Dysbiosis of gut microbiota with reduced trimethylamine-N-oxide level in patients with large-artery atherosclerotic stroke or transient ischemic attack. J Am Heart Assoc. 2020;4:e02699.
15. Collins HL, Drazul-Schrader D, Sulpizio AC, et al. L-carnitine intake and high trimethylamine $\mathrm{N}$-oxide plasma levels correlate with low aortic lesions in ApoE(-/-) transgenic mice expressing CETP. Atherosclerosis. 2016;244:29-37.

16. Landfald B, Valeur J, Berstad A, Raa J. Microbial trimethylamineN-oxide as a disease marker: something fishy? Microb Ecol Health Dis. 2017;28:1327309.

17. Ashar FN, Zhang Y, Longchamps RJ, et al. Association of mitochondrial DNA copy number with cardiovascular disease. JAMA Cardiol. 2017;2:1247-1255.

18. Melser S, Lavie J, Bénard G. Mitochondrial degradation and energy metabolism. Biochim Biophys Acta. 2015;1853:2812-2821.

19. Malik AN, Czajka A. Is mitochondrial DNA content a potential biomarker of mitochondrial dysfunction? Mitochondrion. 2013;13:481-492.

20. Clay Montier LL, Deng JJ, Bai Y. Number matters: control of mammalian mitochondrial DNA copy number. J Genet Genomics. 2009;36:125-131.

21. Torres JAA. The mitochondrial DNA copy number used as biomarker. Int J Mol Biol Open Access. 2018;3:117-119.

22. BordoniL, GabbianelliR.MitochondrialDNA and Neurodegeneration: Any Role for Dietary Antioxidants? Antioxidants. 2020;9(8):764.

23. Castellani CA, Longchamps RJ, Sun J, Guallar E, Arking DE. Thinking outside the nucleus: mitochondrial DNA copy number in health and disease. Mitochondrion. 2020;53:214-223.

24. Yue P, Jing S, Liu L, et al. Association between mitochondrial DNA copy number and cardiovascular disease: current evidence based on a systematic review and meta-analysis. PLOS ONE. 2018;13:e0206003.

25. Al Amir Dache Z, Otandault A, Tanos R, et al. Blood contains circulating cell-free respiratory competent mitochondria. FASEB J. 2020;34:3616-3630.

26. Vallance HD, Koochin A, Branov J, et al. Marked elevation in plasma trimethylamine-N-oxide (TMAO) in patients with mitochondrial disorders treated with oral 1-carnitine. Mol Genet Metab Reports. 2018;15:130-133.

27. Schneider J, Girreser U, Havemeyer A, Bittner F, Clement B. Detoxification of trimethylamine N-oxide by the mitochondrial amidoxime reducing component mARC. Chem Res Toxicol. 2018;31:447-453.

28. Makrecka-Kuka M, Volska K, Antone U, et al. Trimethylamine $\mathrm{N}$-oxide impairs pyruvate and fatty acid oxidation in cardiac mitochondria. Toxicol Lett. 2017;267:32-38.

29. Chen M-L, Zhu X-H, Ran L, Lang H-D, Yi L, Mi M-T. Trimethylamine-N-oxide induces vascular inflammation by activating the NLRP3 inflammasome through the SIRT3-SOD2mtROS signaling pathway. J Am Heart Assoc. 2017;6(9):e006347.

30. Fuchs FD, Whelton PK. High blood pressure and cardiovascular disease. Hypertens. 2020;75:285-292.

31. Leon BM, Maddox TM. Diabetes and cardiovascular disease: epidemiology, biological mechanisms, treatment recommendations and future research. World J Diabetes. 2015;6:1246-1258.

32. Barbara M, David B. Smoking and cardiovascular disease. Arterioscler Thromb Vasc Biol. 2014;34:509-515.

33. Khan SS, Ning H, Wilkins JT, et al. Association of body mass index with lifetime risk of cardiovascular disease and compression of morbidity. JAMA Cardiol. 2018;3:280-287.

34. Bordoni L, Samulak JJ, Sawicka AK, et al. Trimethylamine Noxide and the reverse cholesterol transport in cardiovascular disease: a cross-sectional study. Sci Rep. 2020;10:18675. 
35. Hoff J, Wehner W, Nambi V. Troponin in cardiovascular disease prevention: updates and future direction. Curr Atheroscler Rep. 2016;18:12.

36. Garg P, Morris P, Fazlanie AL, et al. Cardiac biomarkers of acute coronary syndrome: from history to high-sensitivity cardiac troponin. Intern Emerg Med. 2017;12:147-155.

37. Calderón JLM, Pérez JMV, Bustos F, Gómez JCS. Performance characteristics of loci method for measuring cardiac troponin I on the dimension EXL. Pract Lab Med. 2015;1:42-47.

38. Cockcroft DW, Gault MH. Prediction of creatinine clearance from serum creatinine. Nephron. 1976;16:31-41.

39. Fazzini F, Schöpf B, Blatzer M, et al. Plasmid-normalized quantification of relative mitochondrial DNA copy number. Sci Rep. 2018;8:15347.

40. Schmittgen TD, Livak KJ. Analyzing real-time PCR data by the comparative C(T) method. Nat Protoc. 2008;3:1101-1108.

41. Mancini A, Vito L, Marcelli E, et al. Machine learning models predicting multidrug resistant urinary tract infections using "DsaaS". BMC Bioinformatics. 2020;21:347.

42. Velasquez MT, Ramezani A, Manal A, Raj DS. Trimethylamine N-oxide: the good, the bad and the unknown. Toxins. 2016;8(11):326.

43. Silva FSG, Simoes RF, Couto R, Oliveira PJ. Targeting mitochondria in cardiovascular diseases. Curr Pharm Des. 2016;22: 5698-5717.

44. Koller A, Fazzini F, Lamina C, et al. Mitochondrial DNA copy number is associated with all-cause mortality and cardiovascular events in patients with peripheral arterial disease. J Intern Med. 2020;287:569-579.

45. Mengel-From J, Thinggaard M, Dalgård C, Kyvik KO, Christensen $\mathrm{K}$, Christiansen L. Mitochondrial DNA copy number in peripheral blood cells declines with age and is associated with general health among elderly. Hum Genet. 2014;133:1149-1159.

46. Yidan G, Liufu C, Pengpeng Y, Junjuan L, Shouling W, Yang L. Change of kidney function is associated with all-cause mortality and cardiovascular diseases: results from the Kailuan study. $J$ Am Heart Assoc. 2018;7:e010596.

47. Manjunath G, Tighiouart H, Coresh J, et al. Level of kidney function as a risk factor for cardiovascular outcomes in the elderly. Kidney Int. 2003;63:1121-1129.

48. Flint AC, Conell C, Ren X, et al. Effect of systolic and diastolic blood pressure on cardiovascular outcomes. $N$ Engl J Med. 2019;381:243-251

49. Prestes PR, Charchar FJ. Is there a link between mitochondrial DNA and blood pressure? J Hum Hypertens. 2017;31:761-762.

50. Dikalov SI, Ungvari Z. Role of mitochondrial oxidative stress in hypertension. Am J Physiol Heart Circ Physiol. 2013;305:H1417 -H1427.

51. Lei L, Guo J, Shi X, et al. Mitochondrial DNA copy number in peripheral blood cell and hypertension risk among mining workers: a case-control study in Chinese coal miners. J Hum Hypertens. 2017;31:585-590

52. Eirin A, Saad A, Tang H, et al. Urinary mitochondrial DNA copy number identifies chronic renal injury in hypertensive patients. Hypertens. 2016;68:401-410.

53. Eirin A, Saad A, Woollard JR, et al. Glomerular hyperfiltration in obese African American hypertensive patients is associated with elevated urinary mitochondrial-DNA copy number. Am J Hypertens. 2017;30:1112-1119.
54. Whitaker RM, Stallons LJ, Kneff JE, et al. Urinary mitochondrial DNA is a biomarker of mitochondrial disruption and renal dysfunction in acute kidney injury. Kidney Int. 2015;88:1336-1344.

55. Tin A, Grams ME, Ashar FN, et al. Association between mitochondrial DNA copy number in peripheral blood and incident CKD in the atherosclerosis risk in communities study. J Am Soc Nephrol. 2016;27:2467-2473.

56. Zhan M, Brooks C, Liu F, Sun L, Dong Z. Mitochondrial dynamics: regulatory mechanisms and emerging role in renal pathophysiology. Kidney Int. 2013;83:568-581.

57. Small DM, Coombes JS, Bennett N, Johnson DW, Gobe GC Oxidative stress, anti-oxidant therapies and chronic kidney disease. Nephrology. 2012;17:311-321.

58. Che R, Yuan Y, Huang S, Zhang A. Mitochondrial dysfunction in the pathophysiology of renal diseases. Am J Physiol Renal Physiol. 2014;306:F367-F378

59. Navarro-González JF, Mora-Fernández C, Muros de Fuentes M, García-Pérez J. Inflammatory molecules and pathways in the pathogenesis of diabetic nephropathy. Nat Rev Nephrol. 2011;7:327-340.

60. Wong J, McLennan SV, Molyneaux L, Min D, Twigg SM, Yue DK. Mitochondrial DNA content in peripheral blood monocytes: relationship with age of diabetes onsetand diabetic complications. Diabetologia. 2009;52:1953-1961.

61. Xu FX, Zhou X, Shen F, Pang R, Liu SM. Decreased peripheral blood mitochondrial DNA content is related to $\mathrm{HbA} 1 \mathrm{c}$, fasting plasma glucose level and age of onset in type 2 diabetes mellitus. Diabet Med. 2012;29:e47-e54.

62. Fazzini F, Lamina C, Raftopoulou A, et al. Association of mitochondrial DNA copy number with metabolic syndrome and type 2 diabetes in 14176 individuals. J Intern Med. 2021. https://doi. org/10.1111/joim.13242

63. Wei R, Ni Y, Bazeley P, et al. Mitochondrial DNA content is linked to cardiovascular disease patient phenotypes. J Am Heart Assoc. 2021;10:e018776

64. Jaworska K, Hering D, Mosieniak G, et al. TMA, a forgotten uremic toxin, but not TMAO, is involved in cardiovascular pathology. Toxins. 2019;11(9):490

65. Jaworska K, Bielinska K, Gawrys-Kopczynska M, Ufnal M. TMA (trimethylamine), but not its oxide TMAO (trimethylamineoxide), exerts haemodynamic effects: implications for interpretation of cardiovascular actions of gut microbiome. Cardiovasc Res. 2019;115:1948-1949.

66. Restini CBA, Fink GD, Watts SW. Vascular reactivity stimulated by TMA and TMAO: Are perivascular adipose tissue and endothelium involved? Pharmacol Res. 2020;163:105273.

67. Era S, Kuwata K, Imai H, Nakamura K, Hayashi T, Sogami M. Age-related change in redox state of human serum albumin. Biochim Biophys Acta. 1995;1247:12-16.

68. Bito R, Hino S, Baba A, Tanaka M, Watabe H, Kawabata H. Degradation of oxidative stress-induced denatured albumin in rat liver endothelial cells. Am J Physiol Cell Physiol. 2005;289:C531-C542.

69. Rahmani-Kukia N, Abbasi A, Pakravan N, Hassan ZM Measurement of oxidized albumin: An opportunity for diagnoses or treatment of COVID-19. Bioorg Chem. 2020;105:104429.

70. Lee HC, Wei YH. Mitochondrial role in life and death of the cell. $J$ Biomed Sci. 2000;7:2-15.

71. Cayci T, Kurt YG, Akgul EO, Kurt B. Does mtDNA copy number mean mitochondrial abundance? J Assist Reprod Genet. 2012;29:855 
72. Qiu C, Hevner K, Abetew D, et al. Mitochondrial DNA copy number and oxidative DNA damage in placental tissues from gestational diabetes and control pregnancies: a pilot study. Clin Lab. 2013;59:655-660.

73. Liu C-S, Tsai C-S, Kuo C-L, et al. Oxidative stress-related alteration of the copy number of mitochondrial DNA in human leukocytes. Free Radic. Res. 2003;37:1307-1317.

74. Lin C-S, Wang L-S, Tsai C-M, Wei Y-H. Low copy number and low oxidative damage of mitochondrial DNA are associated with tumor progression in lung cancer tissues after neoadjuvant chemotherapy. Interact Cardiovasc Thorac Surg. 2008;7:954-958.

75. Mercer JR, Cheng K-K, Figg N, et al. DNA damage links mitochondrial dysfunction to atherosclerosis and the metabolic syndrome. Circ Res. 2010;107:1021-1031.

76. Yu E, Calvert PA, Mercer JR, et al. Mitochondrial DNA damage can promote atherosclerosis independently of reactive oxygen species through effects on smooth muscle cells and monocytes and correlates with higher-risk plaques in humans. Circulation. 2013;128:702-712.

77. Picard M, Turnbull DM. Linking the metabolic state and mitochondrial DNA in chronic disease, health, and aging. Diabetes. 2013;62:672-678.

78. Révész D, Verhoeven JE, Picard M, et al. Associations between cellular aging markers and metabolic syndrome: findings from the CARDIA study. J Clin Endocrinol Metab. 2018;103:148-157.

79. Huang C-H, Su S-L, Hsieh M-C, et al. Depleted leukocyte mitochondrial DNA copy number in metabolic syndrome. J Atheroscler Thromb. 2011;18:867-873.

80. Hang D, Nan H, Kværner AS, et al. Longitudinal associations of lifetime adiposity with leukocyte telomere length and mitochondrial DNA copy number. Eur J Epidemiol. 2018;33:485-495.
81. Carugno M, Pesatori AC, Dioni L, et al. Increased mitochondrial DNA copy number in occupations associated with low-dose benzene exposure. Environ Health Perspect. 2012;120:210-215.

82. Hou L, Zhang X, Dioni L, et al. Inhalable particulate matter and mitochondrial DNA copy number in highly exposed individuals in Beijing, China: a repeated-measure study. Part. Fibre Toxicol. 2013;10:17.

83. Hou L, Zhu Z-Z, Zhang X, et al. Airborne particulate matter and mitochondrial damage: a cross-sectional study. Environ Health. 2010;9:48.

84. Cioffi F, Senese R, Lasala P, et al. Fructose-rich diet affects mitochondrial DNA damage and repair in rats. Nutrients. 2017;9:323.

85. Bai Y, Carrillo JA, Li Y, He Y, Song J. Diet induced the change of mtDNA copy number and metabolism in Angus cattle. J Anim Sci Biotechnol. 2020;11:84.

86. Hernández-Ríos R, Hernández-Estrada S, Cruz-Robles D, et al. Low fructose and low salt diets increase mitochondrial DNA in white blood cells of overweight subjects. Exp Clin Endocrinol Diabetes. 2013;121:535-538.

\section{SUPPORTING INFORMATION}

Additional Supporting Information may be found online in the Supporting Information section.

How to cite this article: Bordoni L, Petracci I, Pelikant-Malecka I, et al. Mitochondrial DNA copy number and trimethylamine levels in the blood: New insights on cardiovascular disease biomarkers. The FASEB Journal. 2021;35:e21694. https://doi. org/10.1096/fj.202100056R 